\title{
Ambivalenzen und Grenzen des Mitleids bei Jean-Jacques Rousseau*
}

Obwohl Rousseaus Mitleidsbegriff in heutige Verständnisweisen des Mitleids eingegangen ist, spielt er in ihren Thematisierungen nur eine eher untergeordnete Rolle. Rousseaus Beitrag zum modernen Begriffsverständnis steht einerseits im Schatten des Einflusses anderer ethischer Gefühlsauffassungen. $\mathrm{Zu}$ denken ist hierbei an Arthur Schopenhauers Mitleidsethik, auf die sich Autoren wie Walter Schulz oder Ursula Wolf, die das Mitleid zu den Zentralbegriffen ihrer Ethik rechnen, vornehmlich beziehen, an utilitaristische Mitleidskonzeptionen oder an Anknüpfungen an die englische Moral-Sense-Philosophy von David Hume und Adam Smith. Andererseits liegen Ursachen für die periphere Stellung des Begriffes darin, dass er in Rousseaus Werk selbst nur an wenigen Stellen erörtert wird. Die Rezeption dieser Passagen hat sich zudem auf werkimmanente Interpretationen der Bedeutung des Mitleids für Rousseaus Anthropologie und Kulturkritik konzentriert und kaum den Kreis der Philosophiegeschichtsschreibung verlassen.

Mit der mangelnden Aufmerksamkeit auf Rousseaus Begriff entgehen dem Mitleidsdiskurs auch dessen Vorzüge. Im Gegensatz etwa zu Schopenhauer begeht Rousseau nicht den Fehler, das Mitleid zum alleinigen Prinzip seiner Ethik zu erheben. Mitleid steht bei ihm vielmehr Prinzipien der Selbstbezüglichkeit gegenüber, die ein dem vieldiskutierten Schema von Altruismus und Egoismus verwandtes System begründen. Wichtiger noch ist die von Rousseau hervorgehobene Differenz von Natur und Kultur, auf deren Diskussion seine Philosophie vielleicht überhaupt abzielt und die alle Bestimmungen des Mitleids durchzieht. Die Vernachlässigung dieser begrifflichen Unterscheidung im gegenwärtigen Mitleidsdiskurs spiegelt sich in der ungeklärten Beziehung zwischen naturalistischen und kulturalistischen Positionen. ${ }^{1}$ Rousseau gelingt keine * Eine französische Ûbersetzung des Textes erscheint in Éliane Escoubas/László Tengelyi (Hg.),
L'affect et l'affectivité de la philosophie moderne à la phénoménologie, Paris 2007 .

1 Naturalistische Positionen, die ausschließlich die physischen Bedingungen des Mitleids thematisieren, haben in den Naturwissenschaften ungleich stärkere Bedeutung als kulturalistische Positionen, 
befriedigende Aufklärung des Spannungsfeldes zwischen vermeintlich kulturell invarianten und vermeintlich kulturell veränderlichen Eigenschaften des Mitleids, aber er erweitert die philosophische Begriffsbildung durch die Problematisierung der polaren Eigenschaftsbeziehungen.

Mitleid geht bei Rousseau aus einer angeborenen Disposition hervor, die beim Menschen gefördert oder überformt, das heißt auch unterdrückt werden kann. ${ }^{2}$ Ihre kulturelle Ausbildung steht unter Leitung der Vernunft und bewegt sich im Rahmen der Aktivierung der Einbildungskraft, die nicht präsentes Leiden zu imaginieren vermag, sowie der Urteilskraft, die die Leidenssituation und ihre Bedeutung für die mitleidende Person bewertet. Diese kognitiven Komponenten heben Rousseaus Theorie von heute gängigen, auf reine Empfindungsfähigkeit reduzierten Mitleidstheorien ab. ${ }^{3}$ Rousseaus Interesse gilt der Förderung des Mitleids, um menschliches Leiden nicht nur im Einzelfall durch unmittelbare Hilfe zu lindern, sondern zu beseitigen, soweit es die natürlichen und gesellschaftlichen Bedingungen gestatten. Die Radikalität seiner Konzeption zeigt sich aus der Perspektive der von ihm angestrebten egalitären Gesellschaft: In ihr könnte das Leid auf das $\mathrm{Ma} \beta$, dem der Mensch als empfindungsfähiges Wesen nicht entgehen kann, minimiert sein, und die Reichweite des Mitleids auf den Kreis aller Gesellschaftsmitglieder maximiert sein.

Rechnet man die Klärung der Bedingungen zur Verringerung von Leiden zu den Aufgaben der Ethik und das Mitleid zu den hauptsächlichen Motiven leidvermindernder Handlungen, dann erhält diese Konzeption in einer Moderne, die über wachsende Handlungsoptionen zur Leidensbekämpfung verfügt, eine ernstzunehmende Aktualität. Die ethische Relevanz des Mitleidsphänomens ist allerdings umstritten: Befürworterinnen und Befürwortern von Mitleidsethiken stehen unversöhnlich die von rationalistischen Ethiken gegenüber. Eine angemessene Darstellung und Bewertung dieser komplexen Auseinandersetzung kann und muss hier nicht vorgenommen werden. Ich möchte Rous-

die sich allein auf den symbolischen Gehalt des Mitleids beziehen; umgekehrt verhält: es sich bei geisteswissenschaftlichen Thematisierungen des Mitleids. Zum Spannungsverhältnis zwischen diesen beiden Positionen vgl. Schiemann 2004.

${ }^{2}$ Rousseau begreift die natürlichen Eigenschaften als kulturell nicht zu beseitigen und ihre Unterdrückung als „Entfremdung“. Unterdrückung bzw. Entfremdung heißt, dass die innere und äußere Natur dem Menschen nicht mehr gegenwärtig sind, sich von ihm entfernt haben; er steht seinen eigenen Ursprüngen fremd gegenüber. Rousseaus Bestimmungen der egalitären Gesellschaft lassen sich so interpretieren, dass in ihr alle Entfremdung aufgehoben ist. Der von mir gewählte Ausdruck „Überformung" unterstellt, dass das Mitleid bei aller kulturellen Beeinflussung in seinem natürlichen Kern unverändert bleibt. Kulturell kann das natürliche Mitleid funktionsăquivalent durch andere Formen der Anteilnahme oder leidvermindernder Hilfe wie die „Großmut, die Milde, die Menschlichkeit“ (Rousseau 1984 (frz. zuerst 1755), 147), ,die Güte, (...) das Erbarmen, die Wohltätigkeit" (Rousseau 1971 (frz zuerst 1762), 224) ersetzt werden. Allgemein vertritt das Mitleid nur „im Naturzustand ( ...) die Stelle der Gesetze, der Sitten und der Tugend“ (Rousseau 1984, 151, entspr. 57).

${ }^{3}$ Hierunter fallen hauptsächlich die an Schopenhauers Mitleidsethik orientierten und die utilitaristischen Konzeptionen. 
seau darin folgen, die mitleidende Handlungsmotivation nicht vom Standpunkt eines ethischen Prinzips, sondern in Auseinandersetzung mit ihren realen Bedingungen und Realisierungsmöglichkeiten zu beurteilen. Um die mit der Mitleidsreaktion verbundenen Handlungsbedingungen zu klären, steht die begriffliche Erfassung der emotionalen, kognitiven und voluntativen Elemente des Mitleidsphänomens im Fokus meiner Betrachtungen. Meine These ist, dass Rousseaus Begriff eine für die Moderne kennzeichnende ambivalente Struktur aufweist, die aus der Dominanz des Selbstbezuges resultiert.

Eine positive Anknüpfung ist nur in einer kritischen Auseinandersetzung möglich, welche die begrifflichen Bestimmungen von probllematischen Konsequenzen der Anthropologie Rousseaus zum einen abtrennt und zum anderen partiell korrigiert. Meine Rekonstruktion des Begriffes gliedert sich in drei Teile: Zuerst verorte ich seine Position in Rousseaus Werk und fasse seine Bestimmungen zusammen (1.); dann diskutiere ich seine Ambivalenzen auf der Ebene des individuellen Mitleidserlebens (2.); abschließend nehme ich eine Revision und eine Bewertung des Begriffes vor (3.).

\section{Mitleid bei Rousseau}

Rousseau kommt auf das Mitleid, für das er meist den mit dem deutschen Wort im wesentlichen synonymen Ausdruck ,pitié", seltener das etwas bedeutungsweitere „commisération" und nur vereinzelt "compassion" verwendet, an verstreuten Stellen zu sprechen. Den Erörterungen im Discours sur l'inégalité, ${ }^{4}$ im Émile ${ }^{5}$ und im Essay sur l'origine des langues ${ }^{6}$ kommt dabei zweifellos die größte Bedeutung zu. Die Kontexte dieser zentralen Belegstellen unterscheiden sich signifikant: Die Ausführungen des Discours sur l'inégalité referieren auf den vor alle Zivilisation gesetzten Naturzustand, die des Émile auf die Erziehung eines Knaben inmitten einer bürgerlichen Gesellschaft und die des Essay auf das zwischen Naturzustand und Zivilisation liegende „Goldene Zeitalter" einer in Familien organisierten Gesellschaftsform. ${ }^{7}$ Insbesondere den Ausführungen des Discours und des Émile ist die unvermittelte Einführung des Themas, auf das Rousseau im weiteren Verlauf der beiden Texte nur beiläufig wieder zu sprechen kommt, gemeinsam. ${ }^{8}$

${ }^{4}$ Rousseau (1755). - Im Folgenden beziehe ich mich auf die dt. Ausgabe Rousseau 1984.

${ }^{5}$ Rousseau (1762). - Im Folgenden beziehe ich mich auf die dt. Ausgabe Rousseau 1971.

${ }^{6}$ Rousseau (1781). - Im Folgenden beziehe ich mich auf die dt. Ausgabe Rousseau 1981.

7 Rousseau 1981, 185 und 187.

${ }^{8}$ Nachdem das Mitleid in Discours sur l'inégalité als eine der letzten Bestimmungen des hypothetischen Naturzustandes gleichsam nachgeschoben wird, spieit es bei der Darstellung und Kritik des folgenden Zivilisierungsprozesses keine nennenswerte Rolle mehr; im Émile dient die Ausbildung des natürlichen Mitleidgefühls vor allem der Herauszögerung des ersten Gefühls der Liebe, der danach vorherrschenden und nicht systematisch aus dem Mitleid abgeleiteten Emotion. Diese Formen 
In den divergierenden Kontexten und fehlenden übergreifenden Textbezügen sehe ich die entscheidenden werkimmanenten Bedingungen für die hier nicht näher zu besprechende Interpretationsvielfalt von Rousseaus Mitleidsbegriff. ${ }^{9} \mathrm{Zu}$ den Streitpunkten gehört die Frage, in welchem Umfang ein einheitlicher Mitleidsbegriff bei Rousseau vorliegt. Meine Rekonstruktion unterstellt, dass die zentralen Aussagen zusammenstimmen und die Pointe des Begriffes gerade in der gemeinsamen Geltung dieser Aussagen besteht.

Mitleid nennt Rousseau den „Widerwillen [répugnance], irgendein empfindendes Wesen, und hauptsächlich unsere Mitmenschen, umkommen und leiden zu sehen “. ${ }^{10}$ Diese Definition lässt offen, ob Mitleid den Impuls zur helfenden Handlung mit umfasst. Auch für die weiteren Bestimmungen des Begriffes bei Rousseau ist die damit vorausgesetzte Unbestimmtheit charakteristisch. Ich werde mich demgegenüber dem gegenwärtigen Sprachverständnis anschließen, das den Handlungsimpuls zur Linderung oder Beseitigung des Leidens als Bestandteil des Mitleids auffasst.

In überraschender Nähe zu heutigen wissenschaftlichen Bemühungen sucht Rousseau, auch Verhaltensweisen von höheren Tieren seiner Bestimmung zuzuordnen. "Für die Menschen nimmt er an, dass das Mitleid als Korrektiv der Selbstliebe (amour de soi) bereits im Naturzustand zur Anwendung komme. Den auch unter Zivilisationsbedingungen vorhandenen natürlichen Gehalt des Mitleids setzt er in einen Instinkt, der durch die Aufmerksamkeit auf ein Leiden ausgelöst wird und vorreflexiv unmittelbar zur Unterstützung des leidenden Wesens veranlasst. ${ }^{12}$ So führt die Wahrnehmung des „Brüllens" verängstigter Tiere, ${ }^{13}$ des „Stöhnens“ schmerzgequälter Menschen ${ }^{14}$ allge-

der Nichtthematisierung lassen sich als Aussicht auf eine humane Zukunftsgestaltung deuten, die des ursprünglichen Mitleids nicht mehr bedarf.

9 Rang 1959, 426ff; Masters 1968, 43ff., 136ff.; Derrida 1974 (frz. zuerst 1967), 283ff.; Fetscher 1975, 75ff.; Hedman 1979; Dent 1989, 113ff.; Morgenstern 1996, 55ff.; Cooper 1999, 96ff.; Wingrove 2000, 30ff; Nussbaum 2000; Sturma 2001, $107 \mathrm{ff}$. Die unterschiedlichen Auslegungen der unbestrittenen Vieldeutigkeit des Mitleidsbegriffes weisen eher auf differente als auf uneindeutige oder ambivalente (Wingrove 2000) Bedeutungen hin.

${ }^{10}$ Rousseau 1984, 57. Die Hervorhebungen in den Zitaten stammen von mir.

11 Rousseau 1984, 143. Vgl. das Lemma „Mitleid“ im Lexikon der Biologie (1998): „Beobachtungen sprechen dafür, dass schon Menschenaffen Mitleid zeigen - etwa, wenn ein adultes Schimpansenweibchen seine sterbenskranke Mutter mit Futter versorgt (J. Goodall) oder wenn ein geschickter Gorilla-Silberrücken ein Gruppenmitglied vom Draht von Fallenstellem befreit (D. Fossey). I. Eibl-Eibesfeldt betrachtet menschliches Mitleid auch als das subjektive Korrelat der Tötungshemmung. Evolutionäre Voraussetzungen von Mitleid waren Gefühlsansteckung, Selbstexploration und Perspektivenübernahme".

12 Rousseau 1984, z. B. 151.

13 Rousseau 1984, 143.

14 Rousseau 1971, 228. 
meiner: jedes hilfeflehenden „Schreis der Natur“15 völlig mechanisch zum Unterstützungsbestreben, wenn es nicht durch widerstrebende voluntative Faktoren gehindert wird. Kognitiv ordnet Rousseau dem natürlichen Mitleid eine Identifikation zu, mit der sich das mitleidende Wesen ,,an die Stelle (...) [des Leidenden] versetzt “. ${ }^{16}$ An dieser Identifikation setzt die kulturelle Förderung und Überformung des Mitleids an, deren einzige Grenze darin besteht, dass dem natürlichen Gehalt des Mitleids nicht dauerhaft zuwidergehandelt werden kann.

Während die Selbstliebe ausschließlich der Erhaltung des Individuums dient, erfüllt das Mitleid die Funktion der Erhaltung der menschlichen Art (espèce). ${ }^{17}$ Im Mitleidserleben bringt sich damit ein - in der Rezeption meist unberücksichtigter ${ }^{18}$ - übergreifender Aspekt zur Geltung, der sowohl die Stabilität des Naturzustandes als auch die Entwicklung der menschlichen Zivilisation allererst ermöglicht. ${ }^{19}$ Mitleid ist aber zugleich auch artstrukturierend, wo es Verwandtschaftsbeziehungen oder Sozialverbände bevorzugt, und arttranszendierend, insofern es einige Tiere einbegreift. ${ }^{20}$

Mitleid ist bei Rousseau wie die Selbstliebe ein Gefühl. ${ }^{21}$ Den Begriff des Gefühls (sentiment) definiert er im Zusammenhang mit dem der Vorstellung (idée): Gefühl ist eine subjektiv erlebte Vorstellung, deren Objektbezug untergeordnete Bedeutung hat, während Vorstellung ein Gefühl ist, bei dem die Aufmerksamkeit ungleich stärker dem Objektbezug als dem damit verbundenen eigenen Erleben gilt. ${ }^{22}$ Jedem Gefühl kommt deshalb auch der Charakter einer Vorstellung zu. An diese Beziehung kann die Unterscheidung von emotionalen und kognitiven Elementen des Mitleidsphänomens anschließen: Grob gesprochen, umfasst in heutiger Terminologie das emotionale bzw.

${ }^{15}$ Rousseau 1984, 123. In diesen Schrei legt Rousseau im Discours sur l'inégalité den Ursprung der menschlichen Sprache, die er damit - anders als im Essay sur l'origine des langues - auf eine Mitleidsreaktion zurückführt.

Rousseau 1984, 147.

17 Ebd. 151.

${ }^{18} \mathrm{Zu}$ den Ausnahmen gehört Masters 1968.

19 „Espèce" hat eine ähnliche und ähnlich weite Bedeutung wie das deutsche Wort „Art". Ein Bezug auf biologische Arten findet sich bei Rousseau nicht nur im Kontext des Mitleids. Vgl. z. B. das Vorkommen des Wortes nach den im - sehr nützlichen - Index von Rousseau 1984 verzeichneten Stellen. Das Merkmal der Arterhaltung wird vor allem im Discours sur l'inégalité betont. - Arterhaltung ist ein antidarwinistisches Merkmal des Mitleids und widerspricht als solches der ansonsten strikt nicht teleologisch verfassten Anthropologie Rousseaus, die nicht auf die Vervollkommnung des Menschen abzielt, sondern dem Menschen nu: das Vermögen zur Vervollkommnung, die Perfektibilität, zuschreibt (Rousseau 1984, $103 \mathrm{ff} ., 131 \mathrm{ff}$.).

Der Mensch hat Mitleid mit den Tieren (Rousseau 1971, 223 und, nicht ganz eindeutig, 224), Tiere höherer Arten zeigen verwandte Reaktionen auf das Leiden von Lebewesen der eigenen Art und anderer Arten (Rousseau 1984, 143).

21 Rousseau 1971, 121 und 224.

${ }^{22}$ Rousseau 1971, 305 Anm. Rousseau bleibt damit an der cartesischen Unterscheidung von Empfindung (sentiment) als körperlich-geistigem Mischzustand und Idee (idée) als reinem geistigen Zustand orientiert, verwischt aber ungleich stärker, als dies bei Descartes angelegt ist, die Grenzziehung zwischen beiden Bewusstseinsformen. Zu Descartes' Phänomenologie des Bewusstseins vgl. Schiemann 2004. 
gefühlsmäßige Element die in der Perspektive der ersten Person Singular privilegiert zugänglichen und das kognitive bzw. vorstellungsmäßige Element die in der Perspektive der dritten Person thematisierbaren Phänomene des Mitleids.

Im Gegensatz zur Selbstliebe ist das Mitleid ein ,relatives Gefühl“ (sentiment rela$\left.t_{i f}\right)^{23}$, da es das Individuum nicht aus einem inneren Impuls heraus, sondern infolge seiner Bezugnahme auf andere Individuen ergreift. ${ }^{24}$ Alle Ambivalenzen des Mitleids stellen sich als Variationen dieser Relativität dar. Diese finden sich in Rousseaus Werk nicht expliziert, sondern sind Ergebnis einer kritischen Interpretation. Die Hauptbestandsstücke, an die sie anknüpfen, habe ich mit diesen einführenden Bemerkungen bereits genannt. Thre Stichworte lauten in der Reihenfolge, in der ich auf sie eingehen werde: „Widerwille“, „Identifikation", „Arterhaltung" und „Vorreflexivität".

\section{Ambivalenzen der vier Bestimmungen des Mitleids}

Erstens. Widerwille. Wie sich im Deutschen die Bedeutung des Wortes "Widerwillen" mit denen des „Widerstrebens" und des ,Ekels" bzw. des „Verdrusses" verbindet, so ist der Ausdruck „, répugnance“ im Französischen sinnverwandt mit „antipathie " und „,dégoût". ${ }^{25}$

„Widerstreben“ bezeichnet, so möchte ich behaupten, treffend die Negativität des Unangenehmen im Mitleidsgefühl und der korrelierten Handlungsintention, die sich auf und gegen ein vorhandenes Leiden richtet. Weitergehend lässt sich der Widerwille als Ausdruck eines tief greifenden Uneinverstandenseins mit der Existenz von Leid interpretieren. Durch besonderes Leiden bloß veranlasst, richtet er sich gegen das Vorhandensein von Leid überhaupt. ${ }^{26}$

${ }^{23}$ Rousseau 1971, 224

${ }^{24}$ In seiner Relativităt trifft sich das Mitleid mit dem als „Eigenliebe“ übersetzten „,amour-propre“. Die Eigenliebe tritt in der Zivilisation neben die Selbstliebe, dient wie diese der Erhaltung des Individuums, wirkt aber nicht aus einem inneren Impuls heraus, sondern aus dem Vergleich zwischen Individuen, dem Rousseau teils ablehnend, teils positiv gegenübersteht. Das Verhältnis von Mitleid und Eigenliebe bleibt uneindeutig. Zum einen betont Rousseau, dass man „keines von den Gefühlen, die uns zwingen, uns mit anderen zu vergleichen “, den Regungen des Mitleids beimischen dürfe (Rousseau 1971, 228). Zum anderen leitet er das Mitleid aus der Eigenliebe ab (Rousseau 1971, 261). Zum Verhältnis von Mitleid und Eigenliebe vgl. Dent 1989 und Masters 1968.

25 Deutsche Bedeutung nach Duden (1997) und L. Mackensen (1952). Nach Sachs 1898 sind, antipathie" (Widerwille, Widerstreben, natürl. Abneigung) und „dégoût" (1. Mangel an Esslust; 2. Ekel, Widerwille; 3 . Verdruss, Unannehmlichkeiten) mit ,répugnance" sinnverwandt.

${ }^{26}$ Rang hält weniger den Ausdruck „Widerwillen“ in der Definition des als „Mitleid“ bezeichneten Phänomens als die Charakterisierung dieses Phänomens als Mitleid für problematisch: „Rousseau spricht dem Naturmenschen noch ein zweites Gefühl zu: das Mitleid. Freilich ist der Ausdruck nicht sehr glücklich gewählt; denn es handelt sich nicht so sehr um das Mitgefühl mit einem Leidenden als um den inneren Widerstand gegen alles Leiden und Leidenmachen" (Rang 1959, 133 im Org. hervorgeh.). 
Die Verwandtschaft zu „Ekel“ und „Verdruss" verweist auf Gefühle, die sich beim Anblick von Leiden einzustellen vermögen, jedoch intentionalen Einstellungen entsprechen, die dem heutigen Verständnis von Mitleid entgegengesetzt sind. Wo ein Impuls zur emotionalen Abwendung von einem Leiden mit dem Impuls zur helfenden Hinwendung gleichzeitig auftritt, wird das Mitleid als Teil einer ambivalenten Gefühlsreaktion von vornherein begrenzt. Dass Rousseau diese kornplexe emotionale Situation bereits im Blick hatte, legen seine Beispiele insbesondere im Émile nahe: Um das Mitleid des Zöglings auszubilden, soll er mit „Blut, Wunden, Sichreie[n], Stöhnen“ von Lebenden, besser aber noch mit „Leichname[n]", die einen „Todesangst nachempfinden" lassen, konfrontiert werden. ${ }^{27}$ Auch in weniger extremen Situationen verbindet sich wahrscheinlich beim Menschen die emotionale Hinwendung zum Leiden eines anderen mit einem spontanen Abwendungsimpuls. ${ }^{28}$ Ordnet man das Mitleid einer ambivalenten Gefühlsreaktion zu, erhält im Hinblick auf die Handlungsbedingungen die Frage nach den Einflussmöglichkeiten der widerstrebenden Gefühle auf das Mitleid Bedeutung. Ließe sich beispielsweise - wie Martha C. Nussbaum behauptet - der Mitleidsimpuls fördern, wenn es gelänge, die Intensität solcher Gefühle des Ekels zu mindern $?^{29}$

Warum Rousseau seine Definition des Mitleids in den Kontext einer ambivalenten Gefühlsreaktion stellt, erhellt sich vor dem Hintergrund seiner Anthropologie, die den Menschen als im Grunde asoziales Wesen begreift, dessen einsames Glück durch Intersubjektivität beeinträchtigt wird. Die Anteilnahme eines Individuums am Befinden von anderen Wesen kontrastiert strukturell mit seinem eigenen Wohlbefinden ${ }^{30}$ In dem für Rousseaus Anthropologie grundlegenden dualen System von Selbstliebe und Mitleid dominiert der Selbst- gegenüber dem Fremdbezug.

Dem Primat des Selbstbezuges genügt Rousseaus oberste moralische Norm, anderen Lebewesen kein Leid zuzufügen, außer es sei für die eigene Selbsterhaltung unvermeid-

27 Rousseau 1971, 228f. - Entsprechend fordert Rousseau, dass man dem Zögling, „des Menschen Los von der traurigen Seite zeigt. Man muss es ihn fürchten lehren. (...) Er muss die menschlichen Nöte sehen und fühlen. Erschüttert und erschreckt seine Phantasie". (Rousseau 1971, 225f.).

${ }^{28}$ Das haben psychologische Untersuchungen einer Gruppie von Forscherinnen und Forschern um C. Daniel Batson vor gut zwanzig Jahren belegt: Batson u. a. 1983.

29 Nussbaum 2001, 347ff.

${ }^{30}$ Im Naturzustand sind die menschlichen Individuen untereinander völlig isoliert (Rousseau 1984, 79ff.), die beiden Geschlechter treffen nur zufällig und kurz ausschließlich zum Zweck der Vermehrung zusammen (Rousseau 1984, 157). Unter Zivilisationsbedingungen sehen die noch nicht den schädlichen Wirkungen der Eigenliebe verfallenen Individuen in der Einsamkeit ihr Glücksideal (Rousseau 1971, 213, 222), weil sie allein in ihrem nur selbst erleb- und erfahrbaren Inneren die letzten Reste der Natur finden: „Das Glück ist nicht dlas Vergnügen. Es besteht nicht aus einer vorübergehenden Veränderung der Seele, sondern aus einem beständigen und ganz innerlichen Gefühl, das niemand beurteilen kann, außer wer es fühlt" (Elousseau 1977, 239). Die Eigenliebe verhindert in ihrer Fixierung auf das Urteil der anderen die: Selbstbesinnung. Absolute Selbstbesinnung hat nur ein göttliches Wesen: „Ein wahrhaft glückliches Wesen ist einsam. Gott allein genießt absolutes Glück. Aber wer von uns kann daran denken" (Rousseau 1971, 222). Zur Einsamkeit bei Rousseau vgl. Baczko 1970. 
lich. ${ }^{31}$ Diesem Prinzip verdankt sich die Rechtfertigungsbedürftigkeit jeder Leidenszufügung. Mitleid ist bei Rousseau immer auch Veranlassung, nach den Gründen für das Vorkommen des betreffenden Leidens zu fragen, und beruhigt sich nur durch den Nachweis eines notwendigen Selbsterhaltungsnutzens. Mit dem Verbot von nicht zu rechtfertigender Leidenszufügung tritt neben die bloß reaktive Mitleidseinstellung ein aktives Prinzip der Leidensverhinderung. Ohne Mitleid würden die Individuen aus blinder Selbstliebe andere Wesen schädigen, rücksichtslos gegen das Wohl auch ihrer nächsten Verwandten und den Fortbestand ihrer Art. $^{32}$

Rechtfertigungsbedürftigkeit von Leiden und Leidensverhinderung durch Mitleid gehören zu den Vorzügen von Rousseaus Begriff, die ihm auch ohne Voraussetzung der Dominanz der Selbstliebe zugeschrieben werden können. ${ }^{33}$ Demgegenüber lassen sich nicht alle Eigenschaften, die exklusiv aus dieser Voraussetzung folgen, überzeugend verteidigen. ${ }^{34}$ So führt die übermäßige Fixierung auf den Selbstbezug zur impliziten These, dass er ausschließlich durch die Wahrnehmung des Leidens eines anderen Lebewesens zu durchbrechen sei. ${ }^{35}$ Pointiert gesprochen, wendet sich das im Grunde immer einsame Individuum erst einer anderen Person mitfühlend $\mathrm{zu}$, wenn diese augenscheinlich in Not ist. Umgekehrt ist bei Rousseau das Glück einer anderen Person vor allem Anlass für negative Emotionen. Fremdes Glück rufe eher Neid als Liebe hervor. ${ }^{36}$ Der Mensch könne sich noch nicht einmal in die Lage derer versetzen, die glücklicher als er sind. ${ }^{37}$ Wie aber soll man neidisch sein, wenn man sich die Lage der Glücklichen schon nicht vorstellen kann? Neid, der erst durch die Tätigkeit der Einbildungskraft ermöglicht wird, tritt bei Rousseau bereits zur Verhinderung seiner eigenen Bedingung an. Solche absurden Konsequenzen ließen sich vermutlich vermeiden, wenn man, Max Scheler folgend, Mitfreude als gleichwertige Form der Anteilnahme an die Seite des Mitleids stellen würde ${ }^{38}$ Ich werde abschließend darauf zurückkommen, dass diese Symmetrie von Mitfreude und Mitleid an die Grenzen von Rousseaus Begriff und des modernen Mitleidsverständnisses heranführt.

Zweitens. Identifikation. Die Bestimmungen des Widerwillens könnte man als notwendige, aber nicht hinreichende Bedingung des Mitleids begreifen. Wie ich nun zeigen

31 "Sorge für dein Wohl mit dem geringstmöglichen Schaden für andere" (Rousseau 1984, 151).

32 Die Menschen wären ,mit all ihrer Moral nie etwas anderes als Ungeheuer gewesen (...), wenn die Natur ihnen nicht das Mitleid zur Stütze der Vernunft gegeben hätte" (Rousseau 1984, 147). Zur Arterhaltung und zur Verhinderung des Leidens vgl. Abschnitt 2 Drittens.

${ }^{33}$ Die Rechtfertigungsbedürftigkeit von Leid wäre aus der Annahme der Negativität der Existenz des Leides, die Aufforderung zur Leidensverhinderung aus Rousseaus Annahme einer weitreichenden Handlungsverantwortung des Menschen zu gewinnen.

${ }^{34} \mathrm{Zu}$ den Eigenschaften, die exklusiv aus der vorausgesetzten Dominanz der Selbstliebe folgen und sich verteidigen lassen, rechne ich die ambivalente Struktur des Mitleidsbegriffes.

$35 \mathrm{Vgl}$. Nussbaum 2000.

${ }^{36}$ Rousseau 1971, 222.

37 Rousseau 1971, 224. Das ist die „1. Grundregel“" zum Mitleid im Émile.

38 Scheler 1913. 
möchte, ist die von Rousseau formulierte Eigenschaft der Identifikation für das Mitleid zwar äußerst förderlich, aber weder hinreichend noch notwendig.

„Wir lassen uns zum Mitleid bewegen“, sagt Rousseau, ,[i]indem wir aus uns selbst heraustreten und uns mit dem leidenden Wesen identifizieren. Wir leiden nur in dem Maß, wie wir meinen, dass der andere leidet $(\ldots) .^{39}$ Man bedenke, wie viel erworbene Kenntnisse diese Öbertragung voraussetzt ${ }^{4}$.

Diese Aussagen über die Leistungen der Einbildungs- und Urteilskraft kontrastieren mit dem behaupteten Mitleidsinstinkt, der nach Rousseau das Hilfeverhalten einiger Tiere ganz und des Menschen teilweise determiniert. ${ }^{41}$ Von einer mentalen Übertragungsrelation kann nur beim Menschen gesprochen werden, der sich des kategorialen Unterschiedes zwischen dem Leiden eines anderen Wesens und seinem Mitleiden bewusst ist. ${ }^{42}$

Rousseaus Charakterisierung der Identifikation legt es nahe, sie als Einheit von Einbildungs- und Urteilskraft aufzufassen. Die Leistung der Einbildungskraft lässt sich als Empathie, das nachfühlende Hineinversetzen in die Lage einer anderen Person, verstehen. Indem in diese explorative Tätigkeit fortlaufend Urteilsbildungen eingehen, die die Leidensumstände der leidenden und die Erfahrungen der mitleidenden Person berücksichtigen, bildet sich eine „Vorstellung“" vom Leiden, das Gegenstand des jeweiligen Mitleids ist. ${ }^{43}$ Rousseau spricht vom „Vergleich der Vorstellungen“, aus dem ein „Nachdenken“ über das Leiden hervorgehe. ${ }^{44}$ Die Identifikation bezieht sich demnach auf ein Konstrukt, das die mitleidende Person als realen Zustand einer anderen zuschreibt. Jede Beurteilung der Größe und Ernsthaftigkeit eines Leidens durch die mitleidende Person setzt, so möchte ich hinzufügen, diese Zuschreibung voraus. ${ }^{45}$

Für Rousseau ist die explorative Tätigkeit allerdings mit einer davon unterschiedenen evaluativen verbunden, die die Reichweite der Identifikation drastisch begrenzt: Das

${ }^{39}$ Das entspricht der ,3. Grundregel“ zum Mitleid im Émile: „Das Mitleid, das man mit anderen empfindet, wird nicht nach der Größe ihres Leidens gemessen, sondern nach dem Gefühl, das man den Unglücklichen beimisst" (Rousseau 1971, 226).

40 Rousseau 1981, 186. - Vgl. Rousseau 1984, 147 und Fousseau 1971, 224.

${ }^{41}$ Rousseau unterstellt allerdings auch für das instinktive Mitleidsverhalten der Tiere eine mentale Identifikationsleistung. Rousseau 1984, 147.

${ }^{42}$ An der Identifikationsrelation Rousseaus knüpft Schopenhauers Definition des Mitleids an: Schopenhauer 1979 (zuerst 1840), 106. Vgl. zur Auseinandersetzung um Schopenhauers Mitleidsethik Hauskeller 2001, $218 \mathrm{ff}$.

${ }^{43}$ Ebd. - Dass Gefühle Urteile sind, behaupten einige der analytisch orientierten Theorien der Gefühle. Vgl. Landweer 2002.

${ }^{44}$ Ebd.

45 Größe und Ernsthaftigkeit, die mit der Liste der elementaren Leidensfälle in Abschnitt 2 Drittens klassifiziert werden, stellen zusammen das erste von drei kognitiven Elementen dar, die nach Nussbaum 2001, 306f. traditionell in die Definition des Mitleids eingehen. Die anderen sind die Schuldlosigkeit des Leidenden (vgl. Abschnitt 2 Viertens) und die Möglichkeit, dass die mitleidende Person in eine ăhnlichen Situation gerät wie die leidende (das entspricht der „2. Grundregel ${ }^{2}$ zum Mitleid im Émile, vgl. die nächste Anmerkung). 
mitleidende Wesen bewerte seine Vorstellung nach der Maßgabe, dass es bei anderen nur die Leiden beklage, vor denen es ,selbst nicht sicher zu sein“" glaube. ${ }^{46}$ Das Mitleid ließe sich deshalb in einer Gesellschaft nur entfalten, wenn man die irrige Vorstellung bekämpfe, dass Privilegien einen Schutz vor menschlichem Leid bieten würden. Nur wenn man das Bewusstsein schaffe, dass nicht einmal Könige vor menschlichem Leid sicher sind, könne man das Mitleid fördern. ${ }^{47}$ Diese Auffassung Rousseaus ist seiner einseitigen Ausrichtung auf den Selbstbezug geschuldet und bedarf der Korrektur. Um die ganze Reichweite der möglichen Gegenstände des Mitleids zu erfassen, empfiehlt es sich, im Gegensatz zu Rousseau auch Leiden aufzunehmen, von denen der Mitleidende überzeugt ist, dass sie auf ihn keine Anwendung finden werden. Zum Beispiel mag zwar das Mitleid mit Opfern einer Flutkatastrophe durch den Gedanken an Intensität gewinnen, dass man als Mensch vor Fluten nie sicher ist. Indes muss diese Überlegung keine notwendige Bedingung des Mitleids sein. Der Glaube, nicht selbst in ein konkretes Elend geraten zu können, erschwert vielleicht das Mitleid mit denen, die aktuell in diesem Elend sind, schließt es aber nicht aus. ${ }^{48}$

$\mathrm{Ob}$ aber spezielle Vorstellungen von einem Leiden überhaupt vom Begriff des auf sie bezogenen Mitleids gefordert sind, bleibt grundsätzlich fraglich. Rousseau scheint an anderen Stellen für das Verständnis einer vorreflexiven Mitleidsreaktion vage, unstrukturierte Eindrücke für ausreichend zu halten, wenn er Tieren das Mitleid zul-, aber die Einbildungskraft abspricht. Ein weiteres Argument gegen eine unerlässliche Funktion von Einbildungs- und Urteilskraft würde sich aus dem von ihm behaupteten humanen Mitleid mit leidenden Tieren ergeben, wenn man zeigen könnte, dass sich Menschen in die Situation leidender Tiere nicht hineinzudenken vermögen. In diesem Sinn hat Thomas Nagel in seinem berühmten Aufsatz „What is it like to be a bat?" für die Unmöglichkeit, sich mentale Zustände einer anderen Art vorzustellen, argumentiert. Dass zwischen den Arten dennoch Mitleid vorkommt, ließe sich durch einen Selbstbezug erklären, der Leidensäußerungen anderer Arten in Analogie zur Erfahrung eigener Leiden aufnimmt. Diese Form des Mitleids wäre einer „Gefühlsansteckung“, die sich der

${ }^{46}$ Rousseau 1971, 225. Diese Behauptung bildet die ,2. Grundregel“" zum Mitleid im Émile. Sie lässt sich auch auf Tiere beziehen, da Rousseau ihnen auch Vorstellungen (idées) zuschreibt (Rousseau 1984, 101).

${ }^{47}$ In der Verkennung des elementaren Charakters der menschlichen Leiden (vgl, die Liste in Abschnitt 2 Drittens.) sieht Rousseau das größte Hindernis für die Entfaltung des Mitleids. Die Illusion, dem Leiden als Naturbestimmung des zivilisierten Menschen entgehen zu können, verdanke sich allein dem Besitz gesellschaftlicher Privilegien: „Warum haben Könige kein Mitleid mit ihren Untertanen? Weil sie nie damit rechnen, jemals nur Mensch zu sein" (Rousseau 1971, 225). Angesichts des beeindruckenden medizinisch-technischen Fortschrittes wird man heute nicht mehr ungebrochen nur von Illusionen sprechen können.

48 Ein weniger aktuelles Beispiel wäre das Mitleid des Chors der gemeinen Soldaten mit Philoktet. Sie sind überzeugt, dass sie in seine entsetzliche Lage, die sie sich gut vorstellen können, nicht geraten werden (Sophokles 1968, 169-176). 
Differenz zum Leidenden nicht bewusst ist, verwandt. ${ }^{49}$ Auf die bei ihr fehlende Empathie wäre zurückzuführen, dass es lebensweltlich oft schwer fällt, die Größe und Ernsthaftigkeit des Leidens von Tieren, mit denen man mitleidet, zu beurteilen.

Bisher habe ich zwei Argumente genannt, die dagegen sprechen, die Identifikation mit der leidenden Person als notwendige Bedingung des Mitleids aufzufassen. Sie resultieren aus der Differenz zwischen Mitleid und seinem Gegenstand: Die Differenz kann bewirken, dass es für die mitleidende Person unmöglich ist, vom Gegenstand ihres $\mathrm{Ge}$ fühls eine Vorstellung zu haben. Der Gegenstand kann aber auch so fremdartig sein, dass die mitleidende Person sich von ihm zwar eine lebhafte Vorstellung bildet, von dieser jedoch urteilt, sie treffe nur auf die leidende Person zu. Dass Identifikation auch nicht notwendig zum Mitleid hinführt, entnehme ich wieder der Merkmalsgruppe, die der Einbildungskraft geschuldet ist und die ich als Empathie charakterisiert habe. Empathie ist keine hinreichende Bedingung von Mitleid, weil sie gleichfalls dem humanen Gegenteil, der Grausamkeit, dient. ${ }^{50}$ Die Semantik des Begriffes der Grausamkeit ist mit einem nachfühlend erworbenen Wissen um die Wirkungen der durch die grausame Handlung herbeigeführten Leiden verträglich.

Für den Bereich, in dem Mitleid durch Emıpathie ausgelöst oder gefördert wird, schließt sich an die Feststellung der Differenz von Mitleid und seinem Gegenstand nun eine Ambivalenz von Mitleids- und Glücksempfindung an. Rousseau spricht sie aus, wenn er dem Mitleid die Bezeichnung eines ,süßen“ Gefühls gibt. ${ }^{51}$ Mitleid lege nämlich „Zeugnis von unserem Glück ab", vom Leiden verschont zu sein, ${ }^{52}$ und von der Kraft, die sich im Mitleidenkönnen und Helfen beweist. Das Gefühl der Erleichterung kann sich zwar nur gegenüber den Leiden einstellen, „vor denen man selbst nicht sicher zu sein" glaubt, wächst aber mit der Größe des vorgestellten Leidens. Man kann Rousseau nicht zustimmen, wenn er meint, man empifinde auch dann noch Mitleid, wenn es sich zur „Köstlichkeit“ beim Anblick von fremden Leiden - etwa „eines Unglücklichen, der auf dem Rad stirbt", - steigere. ${ }^{53}$ Vielmehr wird der helfende Handlungsimpuls gerade durch die extreme Differenz von leidender Person und den sie wahrnehmenden Personen blockiert.

49 Den Ausdruck ,Gefühlsansteckung“ übernehme ich von Scheler 1913.

50 Das bemerkt auch Rousseau: Rousseau 1981, 186.

51 Rousseau 1971, 65 und 222; Rousseau 1984, z. B. 1151. - Während Rousseau mit der Wendung „süßes Gefühl" Freude als konstitutiven Bestandteil des Mitleids zu unterstellen scheint, setzt er mitunter die Freude auch dem als Identifikation verstandenen Mitleid entgegen: Wir „versetzen uns zwar an die Stelle des Leidenden, empfinden aber zugleich (...) die Freude, nicht so zu leiden wie er" (Rousseau 1971, 222). Im Gegensatz zum Widervillen, der nur partiell dem Mitleid entgegengesetzt ist, steht Freude dem Leid, zu dem das Mitleid gehört, begrifflich disjunkt gegenüber - ein Gegensatz, der literarisch vielfältig artikuliert wird, z. B. „Glaubst Du, ich kann glücklich sein, wenn ich dich leiden sehe?" (Strindberg 1978 (schwed. zuerst 1902), 39).

52 Rousseau 1971, 232.

53 Brief an Philopolis, in Rousseau 1984, 477. 
Wo diese Selbstverhinderung nicht eintritt, bestätigt sich die Kraft der Verschonten in ihrer helfenden Handlung. Rousseau geht einen Schritt weiter, wenn er als starken Charakter zu kultivieren sucht, mitleiden zu können, ohne seine eigenen, möglichst leidfreien Lebenspläne dabei aufzugeben. ${ }^{54}$ Mit diesem Ansatz liefert er bereits ein Argument gegen Friedrich Nietzsches Auffassung, dass Mitleid eine Distanz zum Elend des Leidens ausschließe, sich notwendig dem Leiden angleiche und die mitleidende Person genauso schwäche, wie die leidende durch ihr Leid gelähmt werde. ${ }^{55}$

Drittens. Erhaltung der Art. Auch bei der dritten Eigenschaft, der „Arterhaltung“, ist die Differenz zwischen Mitleid und seinem Gegenstand thematisch. Während das Mitleid als subjektives Erleben emotional erfahren wird, konstituiert die Realisierung seiner arterhaltenden Funktion bei Rousseau ein objektives, alle Individuen umfassendes Phänomen, das in der Perspektive der dritten Person präsent ist. Dementsprechend nehmen bei ihm unter den Gegenständen des Mitleids die elementaren Leidensvorkommnisse der Gattung den größten Raum ein. Seine Beispiele fügen sich in die seit Aristoteles erstaunlich gleich gebliebene Liste der Mitleidensfälle, von denen ich nennen möchte: Tod, Misshandlungen, Krankheiten, Verletzungen, Alter, Mangel an Nahrung und Freunden, nicht eintretende Erwartungen und Fehlen guter Aussichten. ${ }^{56}$ Rousseaus Anteil an der modernen Begriffsbildung besteht bei der Gegenstandsbestimmung des Mitleids in der Erweiterung dieser Aufzählung um die „moralischen Leiden“, die zwar „vom inneren Schmerz, von Leid, Wehmut und Traurigkeit" herrühren, aber ebenso in der Verhaltensdisposition zu erkennen sind wie die physischen Leiden. ${ }^{57}$ Man muss eine Person nicht kennen, um etwa ihre Niedergeschlagenheit aus ihrem „bleichen Gesicht“, ihrem ,erloschenen, tränenlosen Auge (...) " herauszulesen. ${ }^{58}$

Die bei Rousseau präsente Differenz zwischen subjektivem Erleben und objektivem Gehalt (bzw. zwischen der Perspektive der ersten und der der dritten Person) ist, wie ich meine, für das Mitleid nicht spezifisch, sondern tritt bei allen Gefühlen hervor, die eher auf Sachverhalte als auf Stimmungen referieren. Ich möchte Rousseaus Unterscheidung durch die These zuspitzen, dass die Differenz allerdings kaum weiter auseinander treten kann als bei der konkreten Wahrnehmung eines Leidens mit elementarem Charakter. Den dann bestehenden unvermittelten Kontrast von erlebter Subjektivität und dem kognitiven Bewusstsein über den objektiven Gegenstandsbezug bezeichne ich als Ambivalenz.

Man kann sich die Zwiespältigkeit der divergierenden Bestimmungen am Beispiel des emotionalen und kognitiven Bewusstseins klar machen, das eine mitleidende Person

\footnotetext{
54 Rousseau 1971, 233f., 261.

55 Zum Verhältnis von Rousseau und Nietzsche vgl. Reich 1989.

56 Aristoteles 1995, Kap. II.8, Zeile 1386a6-13; vgl. auch Aristoteles 1985, Kap. III.7 Zeile 1114a2327.

57 Rousseau 1971, 229, - Vgl. Hedman 1979, 435ff.

${ }^{58}$ Ebd.
} 
von einer leidenden hat. Die mitleidende Person fühlt mit der leidenden und betrachtet diese zugleich als bloß konkreten Fall eines allgemeinen Leidens. Wo sich das Mitleid auf das Leiden bestimmter Individuen bezieht, wirkt es - um einen Ausdruck von Walter Schulz zu gebrauchen - „entpersönlichend“ ${ }^{59}$ Dies gilt insbesondere für das Mitleid mit einem nahe stehenden Menschen, dessen vertraute Personalität im Mitleid auf schockierende Weise auf allgemeine Bestimmungen menschlichen Daseins reduziert wird, wenn dieses Leiden zu denjenigen gehört, die jedem Menschen widerfahren können. Bei den widerstrebenden Bestimmungen handelt es sich nicht um eine Ambivalenz von Gefühlen. Sie betreffen nicht die entgegengesetzte emotionale Bewertung des Mitleidsgegenstandes ${ }^{60}$ sondern das Verhältnis des Bewwusstseins seiner Objektivität zu jener Bewertung. Das kognitive Bewusstsein hebt sich dabei nicht nur unvermittelt von der Mitleidsemotion ab, sondern bezieht sich zudem kontrastierend auf das emotionale Gegenüber des Mitleids. In ihrer Distanz zum Träger des konkreten Leidens berührt sich die Entpersönlichung mit der von Rousseau angesprochenen emotionalen Abwendung vom Leiden und der emotionalen Erleichterung, nicht von ihm betroffen zu sein.

Das Mitleid bezieht Rousseau nicht nur auf den Nahbereich der mitleidenden Person, sondern auch auf die ganze Menschheit, womit das Leiden entfernter Individuen und beliebig großer Gemeinschaften in den möglichen Umkreis der Gegenstände des Mitleids einrücken. ${ }^{61}$ Ausgehend vom sozialen Nahbereich als dem natürlichen Ursprung des Mitleids ließe es sich durch die gezielte Eijawicklung der Einbildungskraft und der begrifflichen Vermögen auf immer größere Bereiche ausdehnen. Die naturgemäße Angewiesenheit des Mitleids auf die gegenständliche Wahrnehmung von Leiden würde für seine Ausbildung förderlich bleiben, könnte aber durch andere Formen der Kenntnisnahmen wie sprachliche Informationen über ungesehenes entferntes Leiden ersetzt werden. $^{62}$

Rousseau schwankt zwischen Argumenten für eine Anwendung des Mitleids auf die ganze Menschheit und für seine Beschränkung auf soziale Nahbereiche. ${ }^{63}$ Für die Vergrößerung der Reichweite des Mitleids spreche, dass sich mit ihr die Basis zur Beurteilung der Relevanz eines Leidens verbessere. ${ }^{64}$ Doch die Ausweitung des Mitleids auf

59 Schulz 1972, 750f.

${ }^{60}$ Vgl. Graumann 1971.

${ }^{61}$ Die Menschheit ist als ganze mitleidswürdig, weil es überhaupt elend ist zu leben (Rousseau 1971, 222f.).

62 Vgl. Rousseaus Hinweis auf die Bedeutung von Erzählungen in Rousseau 1971, $238 \mathrm{ff}$.

${ }^{63}$ Die Aufrechterhaltung der Ambivalenz, die mit dem Bezug des Mitleids auf Nahbereiche gegeben ist, vertritt Rousseau vor allem im Kontext seiner politischen Philosophie, wie z. B. im „Discours sur l'économie politique": „Es scheint, dass das Gefühl der Menschenliebe sich verflüchtigt und abschwächt, wenn es sich auf die ganze Erde erstreckt" (Rousseau 1989, 350), vgl. Fetscher 1975, 77. Ein Beispiel für die mit der Anwendung auf die ganze Menschheit gegebene Aufhebung der Ambivalenz bei Rousseau 1971, 261.

${ }^{64}$ Rousseau 1971, 261. Vgl. Abschnitt 2 Viertens. 
die ganze Menschheit führe die Gefahr seiner „Verdünnung“ bei sich. ${ }^{65}$ „Verdünnung“ meint die Verminderung der Relevanz sowohl der unmittelbaren Gegenstandsbezüge wie des subjektiven Erlebens. Für bedenklich hält Rousseau die Verdünnung des Mitleids, weil sie die von ihm in Emotionen gesetzte Handlungsgrundlage untergräbt. Er integriert zwar nicht durchgängig den zur Hilfe bereiten Handlungsimpuls in seinen Mitleidsbegriff, verleiht diesem aber eindrucksvoll eine praktische Dimension. Ein unterstützungswilliges Gefühl wird nach Rousseau zur Heuchelei, sobald es sich nicht in Tätigkeit umsetzen kann, weil seine Gegenstände außerhalb des Handlungsradius der Mitleidenden liegen. Rousseaus Gebot der Übereinstimmung von Fühlen, Denken und Handeln macht ihn zum Pragmatisten des Mitleids. ${ }^{66}$ Der Zweck aller praktischen Bestimmungen des Mitleids liegt dabei in der Arterhaltung. Nicht einzusehen ist jedoch, warum die Motivation zur leidvermindernden Handlung eine emotionale Basis haben muss. ${ }^{67}$ Rousseau ist an anderer Stelle selbst der Auffassung, dass sich das Mitleid durch funktionsäquivalente, nicht notwendig emotionale Handlungsgründe wie Gesetze und Sitten ersetzen lasse. ${ }^{68}$

Viertens. Vorreflexivität. Rousseau begreift die bisher genannten Kennzeichen des Widerwillens, der Identifikation und der Arterhaltung als Naturbestimmungen, das heißt kulturinvariante Elemente des Mitleids. ${ }^{69}$ Nicht nur begeht er hierbei den Fehler, alle Kulturinvarianz für unveränderlich zu halten ${ }^{70}$ sondern er setzt sie irrtümlich auch mit den unmittelbar wirksamen und vorreflexiven, das heißt nicht begrifflich erfassten Ele-

${ }^{65}$ Belege bei Fetscher 1975, $76 \mathrm{f}$.

${ }^{66} \mathrm{Zu}$ diesem Gebot: Starobinski 1988. Der von Rousseau mitbegründeten Kultur der Innerlichkeit entspricht es allerdings, dass Gefühle nicht notwendig äußerlich erkennbaren Ausdruck finden. Beim Mitleid lehnt er jedoch jede moralische Bewertung des Gefuhls ab, die seine leidensvermindernde Realisierung unberücksichtigt lässt. Für die Kritik der bloß reflexiven Einstellung zum Leiden und untätigen Thematisierung des Mitleids ist die Kritik am Philosophen, der sich ,nur die Ohren zuzuhalten und sich ein paar Argumente zurechtzulegen" braucht, paradigmatisch. Ihm stellt Rousseau „die Marktweiber, welche (...) die rechtschaffenen Leute daran hindern, einander umzubringen", gegenúber (Rousseau 1984, 149).

67 „Der Mensch ist (...) nur ein fühlendes Wesen, das einzig und allein seine Leidenschaften beim Handeln befragt, und dem die Vernunft nur dazu dient, um die Dummheiten auszubügeln, die er ihretwegen begeht" (Rousseau 1977, 278).

gl. Anm. 2.

${ }^{69}$ Den hypothetischen Charakter seiner Aussagen über das natürliche Mitleid der Menschen hat Rousseau vermutlich verkannt. Seine Annahme, dass es rein nur im fiktiven Naturzustand vor aller Zivilisation vorkomme (Rousseau 1984, 147ff.), hindert ihn nicht, Reste seiner unmittelbaren Wirksamkeit auch unter den schlimmsten Formen der Vergesellschaftung zu beschwören (Rousseau 1984, 145). Der für die Behauptung der Fortexistenz des Mitleids unter den Zivilisationsbedingungen notwendige Nachweis der Kulturinvarianz seiner behaupteten Naturkomponenten findet sich bei Rousseau nur ansatzweise (Rousseau 1984, 143). In Rousseau 1971, 222 versucht er, das Mitleid als anthropologische Konstante zu begründen.

70 Rousseau 1984, 43ff., 77ff. Fälschlicherweise begreift Rousseau die Natur als überhaupt nicht entwicklungsfähig. 
menten des Mitleids gleich. ${ }^{71} \mathrm{Ob}$ und in welcher Weise sich das Mitleid als Widerfahrnis aufdrängt, verändert sich aber mit den verschiedenen Zeiten und Orten. Einbildungsund Urteilskraft vermögen durch kulturelle Praxen der Einübung und Gewöhnung neue Räume der Unmittelbarkeit im Vorreflexiven zu eröffnen. Rousseau selbst gibt ein Beispiel, wenn er die „moralischen Leiden“" neu in die Liste der Leidensfälle aufnimmt.

Rousseaus Bewertung des natürlichen Mitleids fällt gegensätzlich aus. Einerseits soll man das Gefühl ernst nehmen, wo es sich unmittelbar einstellt und der Reflexion vorangeht. Andererseits weist er daraufhin, dass das Gefühl selbst kein Maß kennt und der Beschränkung durch die Vernunft bedarf, um nicht angesichts des unübersehbaren Leidens auf der Welt ins Uferlose anzuschwellen. ${ }^{72}$ Für die leitende Tätigkeit der Vernunft gibt er im Émile nur die eine Regel an, dass man sich dem Mitleid nach Maßgabe der Gerechtigkeit (justice) überlassen solle, denn diese trage zum Gemeinwohl (commun) der Menschen am meisten bei. ${ }^{73}$ Gerechtigkeit, die jeder Person das ihrer Stelle in der Gemeinschaft Gemäße zukommen lässt, bezeichnet bei Rousseau kein Natur-, sondern ein durch den „Gemeinwillen“ (volonté générale) zu begründendes Gesellschaftsprinzip. ${ }^{74}$ Mit ihrer A.usrichtung an der Gerechtigkeit geraten die Gegenstände des Mitleids in größte Entfernung zur fortbestehenden vorreflexiven Unmittelbarkeit. Denn mit dem Wechsel der Kulturen variiert die Klasse der Leiden, die aus Gerechtigkeitsgründen jeweils für mitleidswürdig gehalten werden. Im abendländischen Kulturkreis fallen hierunter meist nicht die schuldhaft durch die leidende Person selbst verursachten Leiden. ${ }^{75}$ Rousseau entscheidet die Frage der Schuldhaftigkeit pauschal auf der sozialen Ebene von Gruppenzugehörigkeiten: In der Regel würden die Reichen schuldhaft, das Volk bzw. die Armen und Unterdrückten aber schuldlos leiden. ${ }^{76}$ Ein Reicher sei noch nicht einmal beklagenswert, wenn er ,unglücklicher als der letzte Arme“ wäre. 77

Die Unterordnung des Mitleids unter die Gerechtigkeit versagt allerdings, wo sich Mitleid auch gegenüber dem für gerecht gehaltenen Leiden einstellt. Die von Rousseau nicht im Kontext des Mitleids diskutierten Haltungen des Erbarmens, der Gnade bzw. der Milde bewegen sich auf der Grenze zwischen dem Bereich des gerechtig-

71 Rousseau 1984, 143.

72 Der Zögling wird nur äußerst selektiv mit Leiden konfrontiert: „Ein Beispiel, richtig gewählt (,..), wird (...) [Émile] einen Monat lang bewegen" (Rouss.eau 1971, 234).

73 Rousseau 1971, 261.

74 Vgl. Dent 1992 Art. „Justice“. Rousseaus Begriff der Gerechtigkeit ist nicht eindeutig. Seine Fundierung durch das Mitleid führt auf einen anderen kulturinvarianten Begriff.

75 Nussbaum 2001.

${ }^{76}$ Kein Mensch könne dauerhaft vor den elementaren Leiden sicher sein. Gleichwohl seien „Glück und Unglück" nicht „gleichmäßig verteilt": Es leiden die unteren Stände unvergleichbar mehr als die oberen (Rousseau 1971, 227).

77 Rousseau 1971, 227. - Scheler fasst das Beklagen eines Menschen als schwache Form des Mitleids (Scheler 1913, 142). 
keitsgeregelten und eines darüber hinausweisenden Mitleids. ${ }^{78}$ Der Begriff des letzteren folgt bei Rousseau aus dem Verbot der nicht legitimierbaren Leidenszufügung und der arterhaltenden Funktion des Mitleids. Beide Bestimmungen garantieren jeweils einen gerechtigkeitsvorgängigen Bereich der Nichtverletzbarkeit, in dem sie einen Minimalschutz vor physischer und psychischer Verletzung etablieren.

\section{Mitleidsgrenzen}

Meine kritische Rekonstruktion möchte ich mit einer Revision von Rousseaus Bestimmung des Mitleids zusammenfassen, ohne die sich ihre fruchtbaren Aspekte nicht sichern lassen. Die Kennzeichen seines Begriffes, die den Impuls zur helfenden Handlung miteinbegreifen, bezeichnen ein Erleben, das, durch fremdes Leiden veranlasst, unmittelbar mit dem Bestreben verbunden ist, dieses Leiden zu lindern, und außerdem gegen das Bestreben gerichtet ist, anderen über das für die Selbsterhaltung notwendige Maß Leid zuzufügen. Diesem Erleben kommt die Empfindung eines Widerwillens gegen die Wahrnehmung eines fremden Leidens nur als notwendige und die Identifikation mit dem leidenden Wesen nur als förderliche Bedingung zu. Beide Bedingungen sind auch Eigenschaften von Phänomenen, die dem Mitleid entgegengerichtet sind: Der Widerwillen eignet auch dem Ekel und dem Verdruss, die Einbildungskraft fördert auch die Grausamkeit. Eine weitere Revision betrifft die Leiden, vor denen sich das mitleidende Wesen selbst sicher zu sein glaubt. Auch sie können Gegenstand des Erlebens einer mitleidenden Person sein, wenn sie für ihr Wohl bedeutsam sind. ${ }^{79}$

Die von Rousseau diskutierten ambivalenten Mitleidseigenschaften werden mit der revidierten Begriffsbestimmung zu Bedingungen verschiedener Mitleidssituationen. In diesem Sinn lassen sich vier Ambivalenzen unterscheiden: 1. die mitleidende Hinwendung, die sich ambivalent auf eine emotionale Abwendung von dem Leiden, auf das das Mitleid gerichtet ist, bezieht, 2. die Anteilnahme am Leiden, die dem zugleich empfundenen Glück, ihm entkommen zu sein, gegenübersteht, 3. das subjektive Gefühlserleben, das mit den objektiven Gegenständen des Mitleids kontrastiert, und 4. die Reflexion, die den vorreflexiven Affekt gezielt einzusetzen vermag, ohne am Charakter seiner Unmittelbarkeit etwas zu ändern.

Ohne Kenntnis dieser Ambivalenzen lässt sich das Mitleid als Handlungsmotivation zur Leidensverhinderung und -minderung nicht fördern. Zwei der genannten Beispiele möchte ich hervorheben: Zum einen das Vorliegen einer ambivalenten Gefühlssituation, in der das Subjekt seine helfende Handlung nur realisieren kann, wenn es sie erfolgreich gegen den

${ }^{78}$ Rousseau diskutiert diese mitleidsverwandten Gefühle nicht im Kontext des Mitleids, aber er erwähnt sie. Vgl. Rousseau 1984, 147 und Rousseau 1971, 224, Anm. 2. - Erbarmen ist für Scheler die stärkste Form des Mitleids, zur Gnade im Anschluss an Rousseaus Mitleidsbegriff vgl. Nussbaum 2000.

79 Nussbaum 2001, 315ff. Zum Begriff des Wohls vgl. Williams 1972, 84ff. 
Abwehrimpuls durchzusetzen weiß. Ist beispielsweise das Mitleiden von einer Empfindung des Ekels begleitet, kann aus Anteilnahme nur geholfen werden, wenn der Ekel entweder abgeschwächt oder umgangen wird. Das andere Beispiel betrifft die Ambivalenz von Anteilnahme am Leiden und dem Glück, von ihm verschont zu sein. Das Glücksempfinden ist - darin dem Ekel gleich - dem Leiden, das durch die Empfindung des Mitleids hervorgerufen wird, entgegengerichtet. Im Gegensatz zum Ekel lässt es sich aber durch Umdeutung in eine positive Beziehung zum Mitleid setzen. Rousseaus dualistische Anthropologie bietet hierfür zwei Möglichkeiten. Das auf sein eigenes Glück abzielende Individuum mag zur helfenden Handlung durch den Hinweis überzeugt werden, dass es dieses Glücksgefühl bewahren und steigern kann, wenn es sich dem Leiden des anderen Wesens auch tätig zuwendet. Unter der Vorgabe des anderen, an der Arterhaltung ausgerichteten Gutes ließe sich das Glück als Zeichen der Verpflichtung zur Hilfe deuten: Das Glück zeigt der verschonten Person an, dass sie zum Zweck der Gemeinschaftserhaltung denjenigen Wesen helfen muss, die sich selbst nicht mehr helfen können.

Die vier Ambivalenzen bezeichnen Grenzen des Mitleids: emotionale Grenzen in seiner Beziehung zu anderen Affekten (Ekel, Verdrussi, Glücksgefühl), mentale Grenzen durch die Differenz zwischen der Mitleid erlebenden (emotionalen) Perspektive der ersten und auf Leiden aufmerksamen (kognitiven) Perspektive der dritten Person, damit verbundene personale Grenzen in der Beziehung des Mitleidenden auf den Leidenden als Leidenden und schließlich moralische Grenzen in der Beziehung zwischen Fragen der Verletzbarkeit und der Gerechtigkeit.

Die Ambivalenzen sind aber auch selbst begrenzt, insofern sie sich aus den grundsätzlichen Beschränkungen der Dominanz der Selbstliebe ergeben. Die emotionale Anteilnahme, die Rousseau einem Individuum, dessen Glücksideal sich auf die eigene Einsamkeit einengt, zugesteht, bezieht sich ungleich stärker auf das Leiden anderer Wesen als auf deren Freude. Auf das Glück der anderen reagiert es mit Neid, der von ihm selbst als Leid erfahren wird. ${ }^{80}$ Es kennt keine eindeutige, unbedingte Hinwendung zum anderen. Jede Anteilnahme bewegt sich in ambivalenten Beziehungen und deutlichen Grenzen. Im Grunde sieht sich das Individuum nur zur Anteilnahme veranlasst, weil seine eigene individuelle Selbsterhaltung nicht ohne die Erhaltung der Art möglich ist. Nur das Leiden der anderen, nicht aber ihre Freude gefährdet die Art.

Rousseaus Anteil an der modernen Begriffsbildung besteht vor allem in der Individualisierung und Subjektivierung einer universell verstandenen Mitleidsthematik. ${ }^{81}$ Traditionell

${ }^{80}$ Davon, dass Rousseau auch versucht, den Widerwillen, fremdes Leiden zu sehen, und die Identifikation mit dem Leidenden aus dem Selbstbezug abzuleiten (z. B. Rousseau 1971, 261), habe ich abgesehen. Deutlich sollte aber geworden sein, dass er die Motivation zum Mitleid in den Selbstbezug legt.

${ }^{81}$ L. Samson charakterisiert die philosophiegeschichtliche Entwicklung des Mitleidsbegriffs als ,zunehmende Betonung und Universalisierung dieses ursprünglich auf Nahsicht eingestellten Verhaltens". Das Identifikationsverhăltnis, das ich neben der Emotionalisierung als Kernpunkt der Subjektivierung betrachte, ist nach Samson eine Innovation des 18. und 19. Jahrhunderts (Samson $1980,1410)$. 
hat sich die philosophische Thematisierung von Gefühlen seit der Antike im polaren Spannungsfeld der angenehm und unangenehm empfundenen bewegt: Lust und Leid, Liebe und Hass, Freude und Schmerz gehören zu den typischen Gegenüberstellungen in Europa. ${ }^{82}$ Rousseau hat dazu beigetragen, das subjektive Erleben als eigentlich angemessene Form der Realisierung angenehmer Gefühle begrifflich auszuzeichnen. Würde man diese Schwerpunktsetzung durch eine entgegengerichtete positive Bewertung intersubjektiver Erlebnisformen aufheben, würde sich vermutlich das gesamte Koordinatensystem auch der Mitleidsthematisierung verändern. Gefühlsreaktionen, die der Anteilnahme am Leiden anderer widerstreben, könnten möglicherweise geringere Berücksichtigung finden, und die Bedeutung des Kontrastes von subjektivem Gefühlsleben und intersubjektiv erfahrbarem Gefühlsgegenstand würde sich eventuell abschwächen. Solche Verschiebungen in der Semantik des Mitleids betreffen die Grundfesten einer ganz auf Individualität und Subjektivität gegründeten Moderne. Wie Rousseau lehrt, muss man an den Elementen gegenwärtiger Selbstverständnisse allerdings nicht gleich rütteln, wenn man das Mitleid als Handlungsmotivation stärken möchte.

\section{Literatur}

Aristoteles (1985), Nikomachische Ethik, übers. v. Eugen Rolfes, Hamburg.

Aristoteles (1995), Rhetorik, übers. v. Franz Günter Sieveke, München.

Baczko, Bronislaw (1970), Rousseau. Einsamkeit und Gemeinschafi, Wien.

Batson, C. Daniel u. a. (1983), ,Influence on Self-Reported Distress and Empathy on Egoistic versus Altruistic Motivation to Help", in Journal of Personality and Social Psycho$\log y 45,706-718$.

Cooper, Laurence D. (1999), Rousseau, Nature, and the Problem of the Good Life, University Park (PA).

Dent, Nicholas J. H. (1989), Rousseau. An Introduction to his Psychological, Social, and Political Theory, Oxford/New York.

Dent, Nicholas J. H. (1992), A Rousseau Dictionary, Oxford.

Derrida, Jacques (1974, frz. zuerst 1967), Grammatologie, Frankfurt a. M. 1983.

Duden (1997), Die sinn- und sachverwandten Wörter, Mannheim.

Fetscher, Iring (1975), Rousseaus politische Philosophie. Zur Geschichte des demokratischen Freiheitsbegriffes, Frankfurt a. M.

Gardiner, Harry M. u. a. (Hg.) (1937), Feelings and Emotion: A History of Theories, New York.

Graumann, Sigrit (1971), Art. „Ambivalenz“, in Joachim Ritter und Karlfried Gründer (Hg.), Historisches Wörterbuch der Philosophie, Darmstadt.

Hauskeller, Michael (2001), Versuch über die Grundlagen der Moral, München.

Hedman, Carl G. (1979), ,Rousseau on Self-Interest, Compassion, and Moral Progress“, in Revue de l'Université d'Ottawa, 49, 430-447.

82 Gardiner 1937. 
Landweer, Hilge (2002), Art. „Gefühle/moraliscthe Gefühle/moral sense", in Christoph Hübenthal u. a. (Hg.), Handbuch Ethik, Stuttgart.

Lexikon der Biologie (1998), Heidelberg.

Mackensen, Lutz (Hg.) (1952), Deutsches Wörterbuch, Stuttgart.

Masters, Rodger D. (1968), The Political Philosophy of Rousseau, Princeton.

Morgenstern, Mira (1996), Rousseau and the Politics of Ambiguity: Self, Culture and Society, University Park.

Nagel, Thomas (1978), The Possibility of Altruism, Princeton.

Nussbaum, Martha C. (2000), „Toleranz, Mitleid und Gnade“, in Rainer Forst (Hg.), Toleranz. Philosophische Grundlagen und gesellschafiliche Praxis einer umstrittenen Tugend, Frankfurt a. M., 144-161.

Nussbaum, Martha C. (2001), Upheavals of Thought. The Intelligence of Emotions, Cambridge.

Rang, Martin (1959), Rousseaus Lehre vom Menschen, Göttingen.

Reich, Klaus (1989), „Rousseau und Kant“, in Rüdiger Bubner u. a. (Hg.), Rousseau und die Folgen, Göttingen, 80-96.

Rousseau, Jean-Jacques (1971, frz. zuerst 1762), Emil oder Über die Erziehung, übers. v. Ludwig Schmidts, Paderborn.

Rousseau, Jean-Jacques (1977), Politische Schriften, übers. v. Ludwig Schmidts. Paderborn.

Rousseau, Jean-Jacques (1981, frz. zuerst 1781), Versuch über den Ursprung der Sprachen, in dem von der Melodie und der musikalischen Nachahmung die Rede ist, übers. v. Hanns Zischler, in ders., Sozialphilosophische und Politische Schriften, München.

Rousseau, Jean-Jacques (1984, frz. zuerst 1755), Diskurs über die Ungleichheit, übers. v. Heinrich Meier, Paderborn.

Rousseau, Jean-Jacques (1989), Kulturkritische und politische Schriften, hrsg. v. Martin Fontius, 2 Bde, Berlin.

Sachs, Karl (1898), Encyklopädisches französisch-deutsches und deutsch-französisches Wörterbuch, Berlin.

Samson, Lothar (1980), Art. „Mitleid“, in Joachim Ritter und Karlfried Gründer (Hg.) Historisches Wörterbuch der Philosophie, Darmstadt.

Scheler, Max (1913), Wesen und Formen der Sympathie, Bonn.

Schiemann, Gregor (2004), „Natur: Kultur und ihr Anderes“, in Friedrich Jäger u. a. (Hg.), Sinn - Kultur - Wissenschaft. Eine interdisziplinäre Bestandsaufnahme, München, 6075 .

Schiemann, Gregor (2005), Natur, Technik, Geist. Kontexte der Natur nach Aristoteles und Descartes in lebensweltlicher und subjektiver Erfahrung, Berlin/New York 2005.

Schopenhauer, Arthur (1979, zuerst 1840), Preisschrift über die Grundlage der Moral, Hamburg.

Schulz, Walter (1972), Philosophie in der veränderien Welt, Stuttgart.

Sophokles (1968), Philoktet, hrsg v. F. C. Görschen, Münster.

Starobinski, Jean (1988), Rousseau. Eine Welt von Widerständen, München/Wien.

Strindberg, August (1978, schwed. zuerst 1902), Ein Traumspiel, Stuttgart.

Sturma, Dieter (2001), Rousseau, München.

Williams, Bernard (1972), Der Begriff der Moral. Eine Einführung in die Ethik, Stuttgart.

Wingrove, Elizabeth R. (2000), Rousseau's Republican Romance, Princeton.

Wolf, Ursula (1984), Das Problem des moralischen Sollens, Berlin/New York.

Wolf, Ursula (1990), Das Tier in der Moral, Frankfurt a. M.

Wolf, Ursula (1993), „Gefühle im Leben und in der Philosophie“, in Hinrich Fink-Eitel und Georg Lohmann (Hg.), Zur Philosophie der Gefïhle, Frankfurt a. M., 112-135. 


\section{Gefühle - \\ Struktur und Funktion}

Herausgegeben

von Hilge Landweer

DEUTSCHE

ZEITSCHRIFT

F ÜR

PHILOSOPHIE

Sonderband

Akademie Verlag 